\title{
AN APPLICATION OF E. H. MOORE'S DETERMINANT OF A HERMITIAN MATRIX*
}

\author{
N. JACOBSON
}

E. H. Moore defined a determinant for any hermitian matrix with elements in a "number system of type $B$. " $\dagger$ In more descriptive language, a system $\Phi$ of this type may be characterized as a quasi-field of characteristic not equal to 2 in which there is defined an involutorial anti-automorphism or involution $a \rightarrow \bar{a}$ :

$$
\overline{a+b}=\bar{a}+\bar{b}, \quad \overline{a b}=b \bar{a}, \quad \overline{\bar{a}}=a,
$$

such that the symmetric elements $(\bar{a}=a)$ are contained in the center. It follows readily that $\Phi$ is either commutative with $\bar{a} \equiv a$, a quadratic field over the field of symmetric elements, or a generalized quaternion algebra over this field. An examination of Moore's theory of determinants shows that it is entirely integral, and hence it is valid if $\Phi$ is any ring with an identity in which there is a unique element $1 / 2$ such that $2(1 / 2) \equiv 1 / 2+1 / 2=1$ and which has an involution $a \rightarrow \bar{a}$ whose symmetric elements are in the center $\Gamma$ of $\Phi$.

The uniqueness of $1 / 2$ implies its symmetry. If $2 a \equiv a+a=0$, then $0=(a+a) / 2=a / 2+a / 2=(1 / 2+1 / 2) a=a$. Let $\Sigma$ and $\mathrm{P}$ respectively denote the sets of symmetric and of skew elements $(\bar{a}=-a)$ of $\Phi$. Then $\Sigma$ and $\mathrm{P}$ are subgroups under the operation + . If $b \varepsilon \Sigma \cap \mathrm{P}$, $b=-b, 2 b=0$, and hence $b=0$. For any $a$ we have

$$
a=\frac{1}{2}(a+\bar{a})+\frac{1}{2}(a-\bar{a})=S a+V a,
$$

where $S a \varepsilon \Sigma, V a \varepsilon \mathrm{P}$. Thus the additive group of $\Phi$ is a direct sum of $\Sigma$ and $\mathrm{P}$. We call $S a$ and $V a$ respectively the scalar and the vector parts of $a$. Now $\Sigma$ is a subring of $\Gamma$, and $\mathrm{P}$ is closed under multiplication by elements in $\Sigma$ and under commutation $[u, v]=u v-v u$. Hence, for any two elements $a, b,[a, b]=[V a, V b] \varepsilon \mathbf{P}$. Thus $S[a, b]=0$ and since, in general, $S(a+b)=S a+S b, S a b=S b a$. Moreover, $a \bar{a}$ and $\bar{a} a$ are symmetric, $a \bar{a}-\bar{a} a$ skew. Hence $a \bar{a}=\bar{a} a$. As usual we call this element, the norm of $a, N a$ and note that $N a b=(N a)(N b)$. Any element $a$ satisfies a quadratic equation with coefficients in $\Sigma$, namely,

$$
x^{2}-(2 S a) x+N a=0 .
$$

* Presented to the Society, February 25, 1939.

$\dagger$ See Moore and Barnard, General Analysis I, American Philosophical Society Publication, chap. 2. We refer to this volume as M-B. 
Thus all the properties noted in M-B, page 103, which do not involve inverses hold in the present case.

We consider the ring $\Phi_{n}$ of $n$-rowed square matrices with elements in $\Phi$. The correspondence $A=\left(a_{i j}\right) \rightarrow \bar{A}^{\prime}=\left(b_{i j}\right)$, where $b_{i j}=\bar{a}_{j i}$ is an involution in $\Phi_{n}$. As is customary, we call the symmetric elements $\left(\bar{A}^{\prime}=A\right)$ under this involution hermitian and we denote their totality as $\mathrm{H}$. If $A, B$ are in $\mathrm{H}, X$ arbitrary, then $A \pm B, A B+B A$ and $\bar{X}^{\prime} A X$ are in $\mathrm{H}$.

Suppose $A=\left(a_{i j}\right)$ is hermitian. If $\sigma$ is a set of distinct indices in the range $1, \cdots, n$ and $f$ is in $\sigma$, we define

$$
s_{\sigma}=\sum(-1)^{r} a_{f h_{1}} a_{h_{1} h_{2}} \cdots a_{h_{r-1} h_{r}} a_{h_{r}},
$$

summed on all permutations of the indices $h_{i}$ of $\sigma$ which are not equal to $f$. It can be shown that $s_{\sigma}$ is in $\Sigma$ and does not depend on the particular index $f$ selected in $\sigma{ }^{*}$ Moore's determinant is defined as

$$
\operatorname{det}_{M} A=\sum s_{\sigma_{1}} s_{\sigma_{2}} \cdots s_{\sigma_{k}},
$$

summed on all partitions of $1, \cdots, n$ into disjoint sets $\sigma_{i}$. The proofs in $M-B$, pages 110-123, are valid for the present case. We require in particular the existence of the adjoint, that is, a certain hermitian matrix $\operatorname{adj}_{M} A$ whose elements are polynomials of $(n-1)$ st degree in those of $A$, such that

$$
A\left(\operatorname{adj}_{M} A\right)=\left(\operatorname{adj}_{M} A\right) A=\left(\operatorname{det}_{M} A\right) 1,
$$

where 1 is the identity matrix. It follows from this as usual that $A$ satisfies its characteristic equation $\operatorname{det}_{M}(x 1-A)=0$ of $n$th degree in $x$ with coefficients in $\Sigma$ that are polynomials in the elements of the matrix $A . \dagger$

We now apply this result to ordinary matrix theory. Let $\Sigma$ be any field of characteristic not equal to $2, \Sigma_{2 n}$ the $2 n$-rowed matrix ring over $\Sigma$ and $Q$ the matrix

$$
Q=\left(\begin{array}{llll}
q & & \\
& q & \\
& & \cdot & \\
& & & q
\end{array}\right), \quad q=\left(\begin{array}{rr}
0 & 1 \\
-1 & 0
\end{array}\right) .
$$

The correspondence $A \rightarrow Q^{-1} A^{\prime} Q$ is an involution in $\Sigma_{2 n}$ and, if

* M-B, p. 114.

$\dagger$ See, for example, Wedderburn, Lectures on Matrices, p. 23, or Albert, Modern Higher Algebra, p. 78. Compare also M-B, p. 128. 


$$
A=\left(\begin{array}{llll}
a_{11} & a_{12} & \cdots & a_{1 n} \\
\cdot & \vdots & \cdots & \vdots \\
\cdot & \cdot & \cdots & a_{n}
\end{array}\right)
$$

where $a_{i j} \varepsilon \Sigma_{2}$,

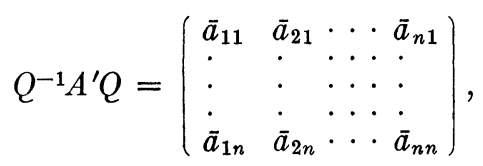

where, in general,

$$
\bar{a}=\left(\begin{array}{rr}
\delta & -\beta \\
-\gamma & \alpha
\end{array}\right) \quad \text { if } \quad a=\left(\begin{array}{ll}
\alpha & \beta \\
\gamma & \delta
\end{array}\right) .
$$

The correspondence $a \rightarrow \bar{a}$ is an involution in $\Phi=\Sigma_{2}$ satisfying the above conditions, and $A \rightarrow Q^{-1} A^{\prime} Q$ is equivalent to $A=\left(a_{i j}\right) \rightarrow \bar{A}^{\prime}$. Hence, if $A$ is symmetric in the sense that $Q^{-1} A^{\prime} Q=A\left(\bar{A}^{\prime}=A\right)$, the above result shows that $A$ satisfies an equation of the $n$th degree in $x$ with coefficients which are polynomials in the elements $\alpha, \beta, \ldots$.

Now suppose $R$ is any nonsingular matrix such that $R^{\prime}=-R$. If $B$ satisfies $R^{-1} B^{\prime} R=B$ and $V$ is any matrix,

$$
\left(V^{\prime} R V\right)^{-1}\left(V^{-1} B V\right)^{\prime}\left(V^{\prime} R V\right)=V^{-1} B V .
$$

We may choose $V$ so that $V^{\prime} R V=Q$ as above.* Thus $A=V^{-1} B V$ is symmetric with respect to the involution $A \rightarrow \bar{A}^{\prime}$. Hence $B$ satisfies an equation $\phi(x ; \alpha, \beta, \cdots)$ of the $n$th degree with coefficients polynomials in the elements of $B$. Let $f(x ; \alpha, \beta, \cdots)$ be the ordinary characteristic polynomial and $\psi(x ; \alpha, \beta, \cdots)$ the minimum polynomial obtained when we regard the elements $\alpha, \beta, \ldots$ in the general matrix satisfying our condition as indeterminates. $\dagger$ As is well known, $f(x)$ and $\phi(x)$ are divisible by $\psi(x)$ and $f(x)$ and $\psi(x)$ have the same irreducible factors.

We now choose $W$ so that

$$
W^{\prime} R W=S=\left(\begin{array}{rc}
0 & 1_{n} \\
-1_{n} & 0
\end{array}\right)
$$

where $1_{n}$ is the identity in $\Sigma_{n}$. Then $C=W^{-1} B W$ satisfies $S^{-1} C^{\prime} S=C$. If

* Wedderburn, p. 96 or Albert, p. 108, loc. cit.

$\dagger$ The conditions on $A=V^{-1} B V$ are $a_{i i}=\alpha_{i i} 1_{2}$ and $a_{j i}=\bar{a}_{i j}, i \neq j$. We regard $\alpha_{i i}$ and the elements of $a_{i j}, i<j$ as indeterminates. If we replace $\Sigma$ by the polynomial ring obtained by adjoining these indeterminates to $\Sigma$, we obtain as before $\phi(B)=0$. 


$$
C=\left(\begin{array}{ll}
L & M \\
N & P
\end{array}\right)
$$

$L, M$, and so on, being in $\Sigma_{n}$, it follows that $M^{\prime}=-M, N^{\prime}=-N$, $P=L^{\prime}$ and conversely any matrix of this form satisfies the condition. If we specialize the indeterminates so that $M=N=0$, we obtain a matrix with irreducible minimum polynomial of degree $n$. It follows that $\psi(x)$ for the general matrix has degree not less than $n$ and hence $\psi(x)=\phi(x)$ and $f(x)=[\phi(x)]^{2}$.

THEOREM. If $B$ is a matrix of $2 n$ rows and columns with elements in a field of characteristic not equal to 2 such that $R^{-1} B^{\prime} R=B$, where $R$ is any non-singular skew symmetric matrix, then the characteristic polynomial $f(x)$ of $B$ has the form $[\phi(x)]^{2}$, where the coefficients of $\phi(x)$ are polynomials in the elements of $B$ and $\phi(B)=0$. If the elements of the general matrix $B$ are regarded as indeterminates, then $\phi(x)$ is irreducible.

University of North Carolina

\section{THE EULER-MACLAURIN SUMMATION FORMULA*}

\section{TOMLINSON FORT}

In this paper an extension of the classical Euler-Maclaurin summation formula $\dagger$ is made to multiple sums. Bernoulli polynomials and numbers of higher order as defined by Nörlund $\ddagger$ enter into the formula and Bernoulli numbers of negative order enter into the proof. Nörlund obtains $\S$ a formula for $\phi(x+\omega)$ in terms of Bernoulli numbers of higher order, and this is called by him an extension of the Euler-Maclaurin formula. His formula permits the ready building up of a simple sum. This is not true, however, of a multiple sum. Steffensen $\|$ calls attention to the fact that a multiple sum can be reduced by summation by parts to a simple sum and the EulerMaclaurin formula for the simple sum used. However, the function to be summed is changed by his suggested transformation and he develops no general formula, nor does he suggest the use of Bernoulli numbers of higher order.

The formula developed in the present paper is equally as easy of

* Presented to the Society, October 29, 1938.

† See, for example, D. Seliwanoff, Lehrbuch der Differenzenrechnung, p. 48, where it is called the Euler sum formula. It is sometimes also called the Maclaurin sum formula. See W. B. Ford, Studies on Divergent Series and Summability.

$\ddagger$ N. E. Nörlund, Differenzenrechnung, p. 129.

\$ Loc. cit., p. 160.

\| J. F. Steffensen, Interpolation, p. 136. 